\title{
Vessels encapsulating tumor clusters in hepatocellular carcinoma: a unique and valuable pathological imaging
}

\author{
Yo-ichi Yamashita, Hideo Baba \\ Department of Gastroenterological Surgery, Graduate School of Life Sciences, Kumamoto University, Chuo-ku, Japan \\ Correspondence to: Yo-ichi Yamashita, MD, PhD, FACS. Department of Gastroenterological Surgery, Graduate School of Life Sciences, Kumamoto \\ University, 1-1-1 Honjo, Chuo-ku, Kumamoto 860-8556, Japan. Email: y-yama@kumamoto-u.ac.jp. \\ Provenance and Peer Review: This article was commissioned by the editorial office, Hepatobiliary Surgery and Nutrition. The article did not undergo \\ external peer review. \\ Comment on: Renne SL, Woo HY, Allegra S, et al. Vessels Encapsulating Tumor Clusters (VETC) Is a Powerful Predictor of Aggressive \\ Hepatocellular Carcinoma. Hepatology 2020;71:183-95.
}

Submitted Oct 25, 2019. Accepted for publication Nov 05, 2019.

doi: 10.21037/hbsn.2019.11.16

View this article at: http://dx.doi.org/10.21037/hbsn.2019.11.16

We read with great interest a paper recently published in Hepatology entitled "VETC (vessels encapsulating tumor clusters) is a powerful predictor of aggressive hepatocellular carcinoma (HCC)" written by Renne et al., a multi-center study from Italy, Korea, and Japan (1). VETC is characterized by the presence of CD34+ vessels completely encapsulating tumor clusters in pathological imaging. The paper reports that VETC is independently associated with early recurrence within 2 years (HR: 1.52 , $\mathrm{P}=0.023$ ), disease-free survival (DFS) [HR: 1.66, $\mathrm{P}=0.002$ ), and overall survivals $(\mathrm{OS})]$ (HR: 2.26, $\mathrm{P}=0.001$ ) in a large cohort of 541 resected HCCs. The term "VETC" first appeared in 2015, in a paper by Fang et al. in Hepatology (2). This earlier study also showed that VETC could predict higher metastasis and recurrence rates of $\mathrm{HCC}$, and that VETC + HCC clusters were released into the bloodstream in an epithelial-mesenchymal transition (EMT)independent manner using a PDX mouse model. The basic characteristics of VETC in HCC were already described in the preceding paper; however, the novelty of this paper is its detailed comparisons of the relationship between VETC and many clinicopathological variables using a large international multi-institutional cohort of resected HCCs with tissue microarray analyses.

The concept of VETC-mediated cancer metastasis is unique. In the classical metastasis model, cancer cells need to invade through the vascular wall and escape anoikis and immune attack. The EMT endows cancer cells with these abilities. In VETC-mediated metastasis, the tissue organization and the encapsulating endothelium protect cancer cells from anoikis and immune assaults. Although the term "VETC" only appeared in 2015, the concept of VETC-mediated metastasis has long attracted attention under a different name. This unique metastasis pattern was first reported in the murine mammary carcinoma model as early as 1993 by Sugino et al. $(3,4)$, who described it as "an invasion-independent pathway of blood-borne metastasis"; they also focused on "tumor nests" surrounded by sinusoidal blood vessels. This pathway is initiated by intravasation of tumor nests enveloped by endothelial cells of sinusoidal vasculature within the tumor. In clinical samples of 10 common human cancers, Sugino et al. also noted the presence of sinusoidal vasculature enveloping tumor nests and of subsequently generated endothelial-covered tumor emboli in efferent veins, especially in HCC, renal cell carcinoma, and follicular thyroid carcinoma (5). We usually consider these three cancers to have similar clinical and molecular characteristics, so their results matched our clinical impressions. In a further study of 80 HCC autopsy specimens, Sugino et al. documented the presence of tumor emboli in liver, lung, and lymphatic routes, consisting of CD31+/laminin + sinusoidal tumor vasculature with basement membrane surrounding HCC nests (6). They named this process "sinusoidal tumor angiogenesis". They did not use the term "VETC"; however, the basic concept of the non-EMT metastasis of VETC + HCC was 
thoroughly described in their variable reports. Therefore, we wish to highlight the contributions of Sugino and his colleagues in this field.

The first report concerning the survival impact of VETC in HCC was published by Ding et al. in 2011 (7) from the same cancer center, (Sun Yat-sen University, China) as Fang et al. (2). They named HCC nests encapsulated by CD34+ endothelium "endothelium-coated tumor clusters (ECTCs)", and ECTCs were an independent poor prognostic factor both in OS (HR: 1.949, $\mathrm{P}=0.001)$ and DFS (HR: 2.085, $\mathrm{P}=0.00002$ ). In addition, ECTCs were associated with micrometastatic endothelium-coated emboli $(\mathrm{P}<0.001)$. Ding et al. showed high proliferation of ECTCassociated emboli using Ki-67 immunohistochemistry, and low apoptosis using TUNEL staining, compared with uncoated emboli. The authors also reported positive correlations between ECTCs and alpha-fetoprotein (AFP) $>25 \mathrm{ng} / \mathrm{mL}(\mathrm{P}=0.033)$, tumor size $>5 \mathrm{~cm}(\mathrm{P}=0.007)$, and grade of tumor differentiation III/IV $(\mathrm{P}=0.024)$. The clinical results of these three papers $(1,2,7)$ concerning VETC in HCC were almost the same; however, the new paper by Renne et al. (1) is variable for several reasons. First, the preceding two papers $(2,7)$ were from a single ethnic population, and patients' characteristics were limited (HBV-related and tumor size $>5 \mathrm{~cm}$ ). This paper includes a more global HCC population with multiple etiologies and relatively small HCC $\leq 5 \mathrm{~cm}$ (82.4\%). In addition, Renne et al. clearly defined the cut-off value of VETC+ as $55 \%$ or more fields using a $\mathrm{K}$-adaptive partitioning algorithm. This cut-off value is very important to widely evaluate the clinical significance of VETC in HCC; therefore, we propose that a validation study using this cut-off value of $55 \%$ should be undertaken using another large cohort. Detailed clinicopathological comparisons between VETC + HCC and VETC - HCC were also performed in this study, and Renne et al. concluded that VETC was significantly associated with several features such as frequent macrotrabecular pattern (Macro-T), less compact pattern (C), less inflammatory infiltrates, and frequent vascular invasion. Finally, they added variable tissue microarray data.

The most important matter is understanding the molecular and pathological meanings of VETC in HCC, and utilizing this unique character in daily medical care of HCC patients. We also reported that histological architecture classification determines the recurrence pattern and prognosis after hepatectomy for HCC (8). Based on pathological architectural classification (9), patients with HCC were classified into four subtypes; microtrabecular (Micro-T), pseudoglandular (PG), Macro-T, and C subtypes; we concluded that Macro-T/ C subtypes had higher AFP levels and early recurrence within 1 year in multiple intrahepatic metastasis $(\geq 4)$ or distant metastasis.

Until we read this paper by Renne et al., we presumed that VETC + HCC and Macro-T HCC have almost the same biological behaviors because of their similar pathological figures and clinical features such as high AFP levels, poorly differentiation, and high rate of microvascular invasion. We also anticipated that Macro-T subtype would demonstrate the higher proliferative capacity of HCC cells more reliably than Micro-T and PG subtypes, and that most Macro-T HCC would display encapsulation by sinusoidal endothelial cells. This paper actually demonstrated a high rate of macro-trabecular massive HCC subtypes (MTM) in VTEC + HCC; however, we were very surprised that MTM showed no survival impact for either OS or DFS. According to Renne et al.'s tissue microarray analyses, VETC + HCC impacted specific molecular subtypes ( $\beta$-catenin/GS+); however, MTM was significantly higher in $\mathrm{p} 53+$ and lower in $\beta$-catenin/GS+. Fang et al. (2) reported that angiopoietin-2 (Ang-2) is essential to VETC formation and metastasis in HCC using orthotopic or subcutaneous Hepa 1-6 xenografts to silence mouse Ang-2. From these important data, we presumed that the difference between VETC + HCC and MTM is caused by the presence or absence of Ang-2 production. The deficiency of Ang-2 in MTM leads to less invasiveness, and has no survival impact. However, we cannot understand the difference in altered gene expression between VETC + HCC ( $\beta$-catenin/GS+) and MTM (p53+). Further studies concerning the molecular difference between VETC+HCC and MTM are needed, and this analysis will require the establishment of a VETC + HCC carcinogenesis model.

Genomic studies in the past decade have elucidated numerous therapeutic targets that can be mapped onto the framework of HCC molecular classification defined by Hoshida et al. (named S1, S2, and S3) (10,11). S1 reflects an aberrant WNT signal pathway, TGF- $\beta$ activation, steatohepatitic HCC, and immune cell infiltrates. S2 is characterized by MYC/AKT activation, Macro-T/C subtypes, high expression of EpCAM, and high AFP levels. $\mathrm{S} 3$ is associated with hepatocyte differentiation (GS+), Micro-T subtype, and good prognosis. In this report by Renne et al., the authors declared that VETC+HCC was classified into $\mathrm{S} 1$; however, according to most clinicopathological characteristics of VETC+HCC in this 
paper, VETC+HCC seems to be classified into S2 as well as Macro-T subtype. The association between VETC+HCC and molecular subclasses remains as a further study issue.

Sorafenib (12) and lenvatinib (13) can be applied to patients with advanced HCC as first-line molecular targeting therapy. Fang et al. also stated that VETC pattern is a predictor of sorafenib benefit in patients with HCC (14). In this report by Renne et al. (1), the authors mentioned that VETC pattern would be easily diagnosed by the biopsy sample; however, we suspect that the discrimination of VETC from MTM patterns in a small sample, even with CD34 immunostaining, would be difficult. Therefore, the efficacy of pretreatment diagnosis of VETC pattern for the choice of drugs for advanced HCC should be prospectively confirmed.

VETC, both an old and a new concept of pathological subclass of HCC, is a unique approach to considering HCC behavior and metastasis. The key to further clarifying molecular mechanisms of VETC in HCC will be the ability to distinguish it from Macro-T pathological subtype, which does not have encapsulating vessels. Nevertheless, we anticipated that utilizing the unique characteristics of VETC in daily medical practices will contribute to improve survival of patients with HCC in the near future.

\section{Acknowledgments}

Funding: None.

\section{Footnote}

Conflicts of Interest: Both authors have completed the ICMJE uniform disclosure form (available at http://dx.doi. org/10.21037/hbsn.2019.11.16). The authors have no conflicts of interest to declare.

Ethical Statement: The authors are accountable for all aspects of the work in ensuring that questions related to the accuracy or integrity of any part of the work are appropriately investigated and resolved.

Open Access Statement: This is an Open Access article distributed in accordance with the Creative Commons Attribution-NonCommercial-NoDerivs 4.0 International License (CC BY-NC-ND 4.0), which permits the noncommercial replication and distribution of the article with the strict proviso that no changes or edits are made and the original work is properly cited (including links to both the formal publication through the relevant DOI and the license). See: https://creativecommons.org/licenses/by-ncnd/4.0/.

\section{References}

1. Renne SL, Woo HY, Allegra S, et al. Vessels Encapsulating Tumor Clusters (VETC) Is a Powerful Predictor of Aggressive Hepatocellular Carcinoma. Hepatology 2020;71:183-95.

2. Fang JH, Zhou HC, Zhang C, et al. A novel vascular pattern promotes metastasis of hepatocellular carcinoma in an epithelial-mesenchymal transition-independent manner. Hepatology 2015;62:452-65.

3. Sugino T, Kawaguchi T, Suzuki T. Sequential process of blood-borne lung metastases of spontaneous mammary carcinoma in C3H mice. Int J Cancer 1993;55:141-7.

4. Sugino T, Kusakabe T, Hoshi N, et al. An invasionindependent pathway of blood-borne metastasis: a new murine mammary tumor model. Am J Pathol 2002;160:1973-80.

5. Sugino T, Yamaguchi T, Ogura G, et al. Morphological evidence for an invasion-independent metastasis pathway exists in multiple human cancers. BMC Med 2004;2:9.

6. Sugino T, Yamaguchi T, Hoshi N, et al. Sinusoidal tumor angiogenesis is a key component in hepatocellular carcinoma metastasis. Clin Exp Metastasis 2008;25:835-41.

7. Ding T, Xu J, Zhang Y, Guo RP, et al. Endotheliumcoated tumor clusters are associated with poor prognosis and micrometastasis of hepatocellular carcinoma after resection. Cancer 2011;117:4878-89.

8. Okabe H, Yoshizumi T, Yamashita YI, et al. Histological architectural classification determines recurrence pattern and prognosis after curative hepatectomy in patients with hepatocellular carcinoma. PLoS One 2018;13:e0203856.

9. Bosman FT, Carneiro F, Hruban RH, et al. The International Agency for Research on Cancer. WHO Classification of Tumours of the Digestive System (IARC WHO Classification of Tumours). 4th ed.; 2010.

10. Hoshida Y, Nijman SM, Kobayashi M, et al. Integrative transcriptome analysis reveals common molecular subclasses of human hepatocellularcarcinoma. Cancer Res 2009;69:7385-92.

11. Tan PS, Nakagawa S, Goossens N, et al. Clinicopathological indices to predict hepatocellular carcinoma molecular classification. Liver Int 2016;36:10818. 
12. Llovet JM, Ricci S, Mazzaferro V, et al. Sorafenib in advanced hepatocellular carcinoma. N Engl J Med 2008;359:378-90.

13. Kudo M, Finn RS, Qin S, et al. Lenvatinib versus sorafenib in first-line treatment of patients with unresectable hepatocellular carcinoma: a randomised phase 3 non- inferiority trial. Lancet 2018;391:1163-73.

14. Fang JH, Xu L, Shang LR, et al. Vessels That Encapsulate Tumor Clusters (VETC) Pattern Is a Predictor of Sorafenib Benefit in Patients with Hepatocellular Carcinoma. Hepatology 2019;70:824-39.

Cite this article as: Yamashita YI, Baba H. Vessels encapsulating tumor clusters in hepatocellular carcinoma: a unique and valuable pathological imaging. HepatoBiliary Surg Nutr 2020;9(4):484-487. doi: 10.21037/hbsn.2019.11.16 